\title{
Assess the Changing Role of Database Technology within Geographic Information System Over the Past 45 Years
}

\author{
Ashna Abdulrahman Kareem Zada* \\ Department of Cartography and Geoinformatics, Faculty of Informatics, Eötvös Loránd University, Hungary
}

\section{${ }^{*}$ Corresponding author:}

Ashna Abdulrahman Kareem Zada,

Department of Cartography

and Geoinformatics, Faculty

of Informatics, Eötvös Loránd

University, Hungary.

E-mail: ashnakareem88@gmail. com

Received: 01 April 2018

Accepted: 04 September 2018

Published: 30 June 2019

\section{DOI}

10.25156/ptj.v9n1y2019.pp26-31

\section{A B S T R A C T}

Spatial data stored in databases have been become progressively crucial in the geographic information system (GIS) field within recent years. Spatial data are increasingly being noted for its significance in representing for real-life relationships. The value of spatial databases cannot be underestimated since it has been established to be an efficient and flexible means of handling enormous spatial datasets. This paper shows the different methods, in which spatial data are integrated by major database vendors and database within GIS. The current report has also examined the new technologies and advancements by database and GIS vendors in relational database management systems in the past four decades, which have facilitated spatial data to become integrated into databases.

Keywords: Database; Evolution; Geographic information system; Spatial data; Technology

\section{INTRODUCTION}

The management and analysis of geographical data are known as geographic information system (GIS). These are the systems through which spatial data gathered from different sources could be stored and interpreted. In the early times, data in GIS were stored in the shape of maps. With the evolution of technology, GIS has evolved and so have its attributes and features. With the passage of time, GIS has adopted different technologies which enabled it to become highly effective and efficient. Throughout the time period of GIS, it can be observed that the system has adopted different technologies, which become the foundation for the modern technology.

The following research report tends to examine the different technologies in terms of database within the GIS. The report presents the details of how the GIS has evolved during the past four decades and has become a significant tool. The report has provided the discussion on the basis of years, hence, summarizing the development and evolution in certain decades. The report has also discussed the various advancements during the considered time period.

\section{DISCUSSION}

\section{Evolution of Database Technology within GIS}

Since its birth in the decade of the 1960s, considerable changes in both expectations and capabilities can be observed. In the lifespan of 45 years, GIS has evolved along with the different technologies that make up the GIS. The role of database technologies has evolved, and it has become the most important attribute of today's successful GIS (Samet, 1990). Moreover, the recent development in the information and communication technology across the globe cannot be neglected, as it has played substantial role in changing the purpose, character of database technologies within the GIS.

Accurate and precise information has always been the cornerstone of effective and timely decision-making. Spatial information, on the other hand, is different from other forms of information. Particularly, two main descriptors are required to complete the information and those are "Where" and "What" (Fisher, 1997). The strong link between the two mentioned descriptors can be observed since long. Conventionally, it involved manually drafted map engaging pen, rub on shading, rulers, dot grids, and other tools to answer the questions. These tools were used to navigate through unfamiliar seas and terrains (Fisher, 1997). Through this, people intended to gather accurate and precise information regarding the location and its physical attributes.

As the time changed, analytical mapped data become an important part of understanding and managing the geographical locations. This shift toward the mapped data is considered as the milestone in the study of GIS (Samet, 1990). 
This works by emphasizing physical description of geographic space, interpreting mapped data, and combining the different layers of map. It is also used to spatially characterizing and communicating complex relationships between the spatial data and information. The foundation for new geospatial concepts and tools is based on the shift toward the map (Fisher, 1997). Through this shift, the descriptors changed to "Where is what (being descriptive)" to "So what and why (prescriptive)" (Samet, 1990). Hence, it can be said that the shift toward the data mapping sets the stage for new geospatial concepts and tools.

With the growing need of accurate information, it was necessary to upgrade the decision-making process. During the era of the 1970s, decision-making relied over mathematical models. These models enabled the researchers to gain quantitative data. Spatial analysis was conducted through manual procedures until the rise of computers and related technologies (Goodchild et al., 1992). Computer enabled researcher to effectively handle voluminous data and efficiently spatial analytical skills. By this viewpoint, GISs are rooted in digital nature in the shape of computerized map.

The evolution and changing role of database technology in GIS can be studied in different manners. Focusing on the past three decades, it is noted that information is key ingredient of any GIS and related activity (Samet, 1990). Gathering accurate and precise information is the key driver for adopting different technologies in the shape of databases (Goodchild et al., 1992).

\section{0s Beginning Years - Computer Mapping}

In the early years of the 1970s, GIS was able to draft maps automatically. This advancement was underpinned by computer mapping ability. The graphical representation of maps lacked quality (Goodchild et al., 1992). The keys in a map including points, lines, and polygons are represented as an organized set of $\mathrm{X}$ and $\mathrm{Y}$ coordinates. Researchers used pen plotters that could redraw the connections at wide range of scales, colors, and projections with the map image (Blum and Calvo, 2000). It provided focused information adequately.

This period is considered as the pioneering work, on which concepts and procedures of modern GIS technology depend (Fisher, 1997). Using computer mapping provided benefits during the editing process.

It could change a portion of map and rapidly redraft the entire portion. It was able to update the resource map in a very short span of time, such as a forest fire burn could be updated in few hours. However, it had some limitations too (Goodchild et al., 1992). The scope of computer mapping was limited due to the radical change in the format of mapped data. Furthermore, Oracle was founded in 1977 and their first database management systems (DBMS) appeared in 1979, but the real development was during the 80s.

\section{0s, Adolescent Years - Spatial Database Management}

The era of the 1980s provided the foundation for DBMS. During this period, computers were used to change the data format. To serve this purpose, spatial DBMSs were developed (Wyatt, 2003). These database technologies were linked with computers with mapping capabilities along with traditional database management capabilities. These systems worked by assigning identification numbers to each geographic feature, for example, timber harvest unit or ownership parcel (Samet, 1990). Through this ability, researchers were able to point at any location and get instant response in the shape of location information. GIS had become so skilled that they could filter the results on the basis of conditions such as soil combination or any forest. A map was displayed as a result.

In the early development of GIS, two different data structures for encoding maps were considered. There was vector data model that was very close to the manual drafting process, in which graphical features of map were represented through lines, in the series of $\mathrm{X}$ and $\mathrm{Y}$ coordinates (Yongli et al., 2008). On the other hand, raster data model was provided with more high-tech mapping technique. It established an imaginary grid over an area. These grids were used to store resource information for each cell on the map. The early consideration wanted to have a universally best structure that could be useful in longer terms (Wyatt, 2003).

By the mid-years of the 1980s, GIS community believed that appropriate data structure depends on the nature of data and the desired processing. GISs were significantly impacted by the realization of duality of mapped data structure (Yongli et al., 2008).

At one end, it was believed that maps with sharp boundaries are best represented as lines. There were certain attributes of map including property ownership, timber sale boundaries, or road networks that could be represented by lines. Others maps such as soils, site index, and slope are interpretation of terrain conditions. The placement of lines representing such conditions is subject to broad classification of continuous spatial data distribution (KumiBoateng and Yakubu, 2010). Hence, from this point of view, the sharp boundary basically represented artificial data based on probability.

Due to the increased demand for mapped data drove the attention of data accuracy, standards, and on its availability. 
During this time, the GIS was also subject to severe data structure issues. Hardware producers, on the other hand, kept their focus on improved digitizing equipment along with manual digital tables, giving automated scanners to facilitate the GIS facilities (Pundt and Runde, 2000).

This gave life to a new industry for map encoding and database design. A market place with demand and supply of digital map products was created (Wyatt, 2003). International, national, and regional organizations began to involve in the standards of digital mapping to ensure better productivity and better information. This era founded the GIS database from project costing to equity investment. Soon, corporate databases were founded. This evolution in the technology was based on the computer technology integrated during the late 1960s (Ian, 2010).

\section{0s, Maturing Years - Map Analysis and Modeling} As GIS kept evolving, the focus shifted from to prescriptive analysis of maps from descriptive query. This was the time when analogous procedures within the GIS were integrated. GIS, during this time, was programmed with mathematical solution to ensure that repeated distance and bearing calculations could be done. This resulted in the effective GIS functionality that mimicked the manual procedures in user's day-to-day activities (Goodchild et al., 1992). These systems also saved energy and time by automating tedious and repetitive operations.

By the middle years of the 1980s, bulk of descriptive query operations was available in many GIS systems. However, this was the period during which attention turned to the comprehensive theory of map analysis. The critical feature of this theory is nature of spatial information (Fekpe, 2003). It was represented numerically, rather than in the analog style, as inked lines on the map. These digital maps are conceptualized as a set of floating maps, having common registration, and permitting the computer to use different digital maps. Through these database technologies, the spatial relationships between the different natures of data could be summarized (Elangovan, 2006). It included database queries and analytical processing skills. Mainly due to the analog representation of traditional map sheets, manual analytical techniques are confined to quantitative processing. Digital representation, on the other hand, represented a wealth of quantitative and qualitative processing possibilities (Blum and Calvo, 2000).

The new theory and its application turned out to be revolutionary. Its application took the shape of two forms, spatial analysis and spatial statistics. This is the time of GIS, in which databases started to take the shape of new and latest GIS systems (Andrews, 2003).
Geophysicists and meteorologists have been using spatial statistics for many years to study the geographic distribution and patterns of mapped data. Through the statistical models, the researchers were able to have more purified information regarding the spatial distribution (Elangovan, 2006). It generated a spatial variation in the data, for example, several plots within a watershed can be used to measure the depth of snow. Conventionally, the data of this nature are analyzed for single value to characterize an entire watershed (Fisher, 1997). The statistics based on spatial data also used measurement and sample location to map relative snow depth in the entire watershed. The traditional non-spatial statistics is the direct extension of numeric-based processing (Fekpe, 2003).

However, spatial analysis applications involved contextbased processing. For example, a forester can assemble the supply of timber by measuring the relative log hauling and skidding accessibility of harvesting parcels. This factor was very useful for wildlife researchers (Ian, 2010). They consider these factors as proximity to roads and relative housing density to map the human activity. This information is incorporated into habitat delineation. At same time, land planners could assess the visual exposure to the various alternative sites. They could view locations, roads, and scenic overlooks (Pundt and Runde, 2000).

In this era, the spatial mathematics also evolved along with spatial statistics on the basis of conventional concepts. The maps were dependent on algebra. The combination of maps and algebra could perform complex map analysis.

The algebra used in this manner is related to the traditional algebra, in which primitive operations including BODMAS are logically sequenced on different variables to form the equations. However, in such maps, they were composed of millions of numbers represented by different variables of the spatial equation (Wyatt, 2003). In this manner, the database constructed was on the basis of mathematical models. As a result, the databases become very effective and could store information better than before (Yongli et al., 2008).

The modern GIS packages comprise traditional mathematical abilities plus an advanced processing system for maps (Ian, 2010). These systems can be used to conduct primary mathematical operations along with complex and advanced mathematical procurers. After all, the new and latest GIS is the combination of numbers.

The new GIS has some similarities as well as some differences with the traditional database technology. In the early times, it expressed by simple automating the existing capabilities; however, its modern form is quite challenging to the nature and utility of maps (Elangovan, 2006). 


\section{0s, Full Cycle Multimedia Mapping}

However with such "improvements developments" in the sector of technology now a days: GIS is not improved "is not effective" rapidly as well. GIS used today comprises of latest technological features, which have enabled the researchers to be highly productive (Goldberg et al., 2009). The database technology is better than before, and therefore, the mapping and spatial data are stored in highly effective manner. The new processing units of GIS are integrated with the global position systems.

They also comprise remote sensing imagery equipment which has enhanced the database technologies (Andrews, 2003). Through global positioning system (GPS) and digital mapping, the entire GISs are squeezed to palm of a hand. The information is processed through highly effective and efficient, databases, enabling in hand information to the researchers and users of GIS. Toggling off and on an aerial photograph provides reality as a backdrop to GIS modeled information (Blum and Calvo, 2000). Moreover, these systems are provided with ancillary systems, including robotics, this enables the GIS to mix and automate the processes and procedures for data collection on various modes.

Apart from the changes in the processing area, the display area of new GIS has evolved, as well. Old graphics including two-dimensional have become old stories, and not spatial information is presented in three-dimensional view of the terrain (Davis, 2001). The information is transformed from virtual reality representing real-life objects including trees, lakes, and buildings on the surface.

\section{0s, GIS on the Web}

Until recently, the only practical method to apply GIS to the issues was to collect all of the necessary parts in one place which was users' desktop. However, today all of the parts, data and software can be accessed remotely.

The GIS Web is a vision for the future because all of the data and application are able to operate together by turning the internet into a massive GIS. Furthermore, it includes real-time feeds of data from sensors and the ability to integrate these with other data to expand their work in terms of having professional results and better analyzing.

In the early days of GIS, data were derived from paper maps and described features on the Earth's surface that was largely static and unchanging. However, thanks to GPS data are being served to users on internet. It is now routine and an increasing amount of real-time data is becoming available.

In the second half of the 1990s, internet has had farreaching impact on the accessibility of GIS data and on the ability of GIS users to share datasets. Rapidly developing technologies allow the user to move away from desktop and as a consequence of use GIS in everywhere. It is important to point out that limited or complementary GIS services are already available in common devices such as mobile phones and increasingly installed easily so it looks to a future bright, in which GIS is progressively mobile and available everywhere.

In addition, a large number of websites offer GIS data for free, sale, and temporary and also provide services that allow users to search for datasets that satisfy certain requirements. For example, OpenStreetMap is one of the many efforts around the world to enlist volunteers in the certain of open free digital maps that are especially important in areas where such maps are not freely available.

Furthermore, the number of available GIServices or GIS web services is growing steadily. GIServices are defined as a program executed at a remote site. The execution of the program is begun remotely by the user. The user may have supplied data or may depend on the data provided by the service or both. For instance, ArcGIS Online is another relatively powerful tool defined by Esri as a "complete, Cloud-Based Mapping Platform," which let users made better (especially thematic) maps. Creating maps from maps published by other users are just one of the many ways to take advantage of the rich collection of data and resources. As well as updating and performing dynamic analysis on GIS data is part of ArcGIS Online, so it will generate smarter maps and reports to provide unique understanding.

Regarding to web map services which refer to more complex and sophisticated geographic data analysis activities than the more common term "Web mapping" such as the Microsoft Virtual Earth and Google Earth platforms. It similarly points to the upcoming of GIS, which after a long majority of desktop applications, now transitioning back to a client-server is planning - whether the client is on a desktop or a mobile application.

Another example, in the past few years, many citizens have become involved in the creation and dissemination of free geographic information in a process known as volunteered geographic information. At the same time, open-source applications have become more and more popular between users. It is a development way for software that joins the power of spread peer review and transparency of procedure. The advantages for using open sources are better quality, higher reliability, more flexibility, and lower cost (or free of cost). Furthermore, the user becomes free to use this type of sources for any purposes, allowing them to adapt it to their specific needs: They can improve the program and released their improvement to the public so the whole 
community can get benefit from it. Another benefit from open sources is that users can access GIS data and analysis tools over the internet. The important point is that GIS software is not required to install it locally and can manipulate maps and data over the web directly. Furthermore, there are many open sources available for Web GIS such as MapServer and GeoServer. It should be mentioned that open sources have not been limited to a specific period of time because it began in the middle of the $90 \mathrm{~s}$ and follows its development to the current days, for example, the popularity of Quantum GIS, Geo tools, Grass GIS, and PostGIS.

All distributed GISs offer massive benefits. For instance, it reduces duplication of effort, allows users to take advantages of remotely located data and services through simple devices, and provides methods of combining information collection through the digital sources. Web GIS offers a vision of a future, in which geographic location is central to a wide range of applications and in which individuals are able to access detailed information such as transportation, weather, and other socially important fields.

\section{Future, Communicating Perceptions, Spatial Reasoning, and Dialogue}

The future of GIS is observed to be quite bright. The database technologies within the GIS will keep evolving (Ian, 2010). The main reason for this is the development in the field of information and communication technology. It is observed that in future, the GIS should comprise cognitive ability. The database technologies should also be based on cognitive skills (Ian, 2010). Moreover, with the growing need of precise information, the information systems should be used to store information beyond mapping, management, and modeling. It should be supported by spatial reasoning and dialogue to find scientific solutions to the problems. In reality, however, there are other factors which are required to be taken care of. It is the social solution. It must use human values, beliefs, judgment, trust, and understanding.

Moreover, these elements are not the usual measures including the quantitative scale, or computer algorithms or traditional decision-making models. From the basis of the system, the effective communication is an essential step and all the parties must be involved in the decision-making process. As a result, the new environment comprises two main elements including consensus building and conflict resolution. On the basis of these two main elements, the GIS and the database information system could be enhanced (Ian, 2010).

\section{Future Challenges and Critical Issues}

Till now, all the technical hurdles around GIS have been tacked over the past decades. GIS applications are on the move, spatial databases are taking comprehensive forms, and there are automation packages available, as well (Kreitz, 2007). However, the biggest challenge for the GIS and its future is the lack of competent users. The biggest hurdle in the GIS development is to educate the masses of potential users on what GIS is and is not. Moreover, it is also required to develop related spatial skills and abilities. For today, it can be said that GIS is not just mapping technology (Kumi-Boateng and Yakubu, 2010). It is much more than this. This has increased the need of personnel, who can be the part of training and development and learning period GIS and its applications. The database technologies in early times used physical store of data and manual processing (Goldberg et al., 2009). After the introduction of computer and its application, much of the data and procedures have been automated (Wyatt, 2003). As a result, the focus of GIS has shifted from being descriptive to prescriptive. In the shifting period, the analytical tools have become highly quantitative. This wealth and ability of processing capabilities have provided opportunities to address complex and spatial issues in new ways.

\section{CONCLUSION}

The above discussion presents the details regarding the technological advancements in databases within the GIS throughout the time. From the above discussion, it can be concluded that since its birth in the year 1960, the GIS has adopted different technologies, and it has become highly effective. With the passage of time, the GIS has not only become effective in terms of data storage, interpretation, and data analysis but also in displaying maps and different features of the unknown terrain.

This advancement in the discipline of GIS is basically driven with the need of accurate and precise information. Today's GIS is highly capable, with automated features, enabling the researchers to have more precise and accurate information regarding the issues. It can be concluded that the role of database has evolved and has become highly critical.

\section{REFERENCES}

Andrews, B. 2003. Techniques for Spatial Analysis and Visualization of Benthic Mapping. Available from: http://www.csc.noaa.gov/ digitalcoast/_pdf/spatial.pdf. [Last accessed on 2012 Dec 21].

Blum, O. and R. Calvo. 2000. Geospatial Data Collection and Analysis as Crucial Processes In an Integrated Census. Available from: http://www.fcsm.gov/01papers/Blum.pdf. [Last accessed on 2012 Dec 21].

Davis, E. 2001. Geographic Information System. Cengage Learning, Canada.

Elangovan, K. 2006. GIS Fundamentals, Applications and Implementations, New India Publishing, India. 
Fekpe, E. 2003. Quality and Accuracy of Positional Data in Transportation. Transportation Research, United Kingdom.

Fisher, P. F. 1997. Editorial: Welcome to the international journal of geographical information science. Int. J. Geogr. Inf. Sci. 11(1): $1-3$.

Goldberg, D. W., J. P. Wilson and C. A. Knoblock. 2009. Extracting geographic features from the internet to automatically build detailed regional gazetteers. Int. J. Geogr. Inf. Sci. 23: 93-128.

Goodchild, M. F., R. Haining and S. Wise. 1992. Integrating GIS and spatial data analysis: Problems and possibilities. Int. J. Geogr. Inf. Syst. 6: 407-423.

Ian, H. 2010. An Introduction to GIS. Pearson Education, India.

Kreitz, M. 2007. Methods for Collecting Spatial Data in Household
Travel Surveys. Available from: http://www.isctsc.let.fr/pastConf/ kruger/10/Kreitz.pdf. [Last accessed on 2012 Oct 30].

Kumi-Boateng. B. and I. Yakubu. 2010. Assessing the quality of spatial data. J. GIS. 43: 507-515.

Pundt, H. and B. Runde. 2000. Visualization of spatial data for field based GIS. Comput. Geosci. 26: 51-56.

Samet, H. 1990. Applications of Spatial Data Structures. AddisonWesley, United States of America.

Wyatt, P. 2003. GIS in Land and Property Management. Taylor and Francis, USA.

Yongli, G., T. Robert and A. Calvin. 2008. Applications of GIS and database technologies to manage a karsts feature database. J. Cave Karst Stud. 68: 144-152. 